\title{
Universiteit
}

Leiden

The Netherlands

\section{Charge detection enables free-electron quantum computation} Beenakker, C.W.J.; Vincenzo, D.P. di; Emary, C.; Kindermann, M.

\section{Citation}

Beenakker, C. W. J., Vincenzo, D. P. di, Emary, C., \& Kindermann, M. (2004). Charge detection enables free-electron quantum computation. Retrieved from https://hdl.handle.net/1887/1294

Version: $\quad$ Not Applicable (or Unknown)

License: $\quad$ Leiden University Non-exclusive license

Downloaded from: https://hdl.handle.net/1887/1294

Note: To cite this publication please use the final published version (if applicable). 


\title{
Charge Detection Enables Free-Electron Quantum Computation
}

\author{
C W J Beenakker, ${ }^{1}$ D P DiVincenzo, ${ }^{2}{ }^{3}$ C Emary, ${ }^{1}$ and M Kinder mann ${ }^{4}$ \\ 'Instituut Lorentz, Universtteit Letden PO Box 95062300 RA Leiden The Netherlands \\ ${ }^{2}$ Department of Nanoscience Delft Universti) of Technology, Lorent:ueg I $2628 \mathrm{CJ}$ Delft The Netherlands \\ ${ }^{3}$ Institute for Theoretical Physics Valckenterstiaat 651018 XE Amsterdam The Netherlands \\ ${ }^{4}$ Department of Phusus Massachusetts Institute of Technology Cambidge Massachusetts 02139 USA
}

(Received 19 Februar y 2004, published 6 July 2004)

\begin{abstract}
It is known that a quantum computer operating on election spin qubits with single election Hamiltonians and assisted by single-spin measuiements can be simulated efficiently on a classical computer We show that the cxponential speedup of quantum algoithms is restored if single-charge measurements ale added These enable the constiuction of a CNOT (contiolled NOT) gate for fiee fermons, using only beam splitters and spin iotations The gate is nearly deterministic if the chatge detector counts the number of elections in a mode, and fully deter munstic if it only measures the panty of that number
\end{abstract}

DOI 10 1103/PhysRevLett 93020501

PACS numbers $0367 \mathrm{Lx} 0367 \mathrm{Pp} 0530 \mathrm{Fk} 7110-\mathrm{w}$

Flying qubits transpoit quantum information between distant memor y nodes and for $\mathrm{m}$ an essential ingredient of a scalable quantum computer [1] Flyıng qubits could be photons [2], but using conduction elections in the solid state for this purpose iemoves the need to convert materal qubits to radiation Since the Coulomb interaction between free elections is strongly scieened, an interaction-fiee mechanism for logical operations on electionic flying qubits could be desirable The search for such a mechanism is stiongly constrained by a no-go theorem $[3,4]$, which states that the exponential speedup of quantum over classical algoithms cannot be ieached with single-election Hamiltonians assisted by single-spin measurements Here we show that the full power of quantum computation is restored if single-charge measurements are added These enable the construction of a CNOT (controlled NOT) gate for fiee fermions, using only beam splitters and spin iotations

The no-go theorem $[3,4]$ applies only to feimions, not to bosons Indeed, in an influential paper [2], Knill, Laflamme, and Milbuin showed that the exponential speedup over a classical algoithm afforded by quantum mechanics can be ieached using only lineai optics with single-photon detectors The detectors interact with the qubits, providing the nonlinearity needed for the computation, but qubil-qubit inteiactions (e g, nonlınear optical elements) are not required in the bosonic case This difference between bosons and fermions explains why the topic of "fiee-election quantum computation" (FEQC) is absent in the literatuie, in contiast to the active topic of "linear optics quantum computation" (LOQC) [5-12] Here we would like to open up the former topic, by demonstrating how the constraint on the efficiency of quantum algor thms for fiee termions can be removed We accomplish this by using the fact that the election callying the qubit in its spin degiee of treedom has also a cha1ge degree of fieedom Spin and charge commute, so a measurement of the charge leaves the spin qubit unaffected To measure the chaige the qubit should interact with a detector, but no qubit-qubit interactions are needed

Charge detectors play a prominent iole in a variety of contexts as which-path detectors they control the visibility of Ahatonov-Bohm oscillations [13], in combination with a beam splitter they piovide a way to entangle two noninteracting paiticles [14], in combination with spindependent tunneling they enable the readout of a spin qubit $[15,16]$ The experimental realization uses the effect of the electirc held of the charge on the conductance of a nearby point contact [17] The effect is weak, because of scieening, but measurable if the point contact is near enough Such a device functions as an electrometer It can count the occupation number of a spatial mode $(0,1$, o1 2 elections with opposite spin) If the point contact is replaced by a quantum dot with a resonant conductance, then it is possible to operate the device as a partity meter It can distinguish occupation number one (when it is on lesonance) from occupation number 0 or two (when it is off iesonance)-but it cannot distinguish between 0 and 2 We will consider both types of chatge detectors in what follows

The general for mulation of fer mionic quantum computation [18] is in teims of local modes which can be either empty of occupied The annihilation operator of a local mode is $a_{l s}$, with spatial mode index $l=1,23$, and spin index $s=\uparrow \downarrow$ For noninteracting fermions the Hamilonian is bilineai in the cieation and annihilation opeiators A local measuiement in the computational basis has projection operators $n_{t s}=a_{t s}^{\dagger} a_{l s}$ and $1-n_{l s}=$ $a_{t s} a_{t s}^{\dagger}$ Terhal and one of the authors [3] showed that the probability of the outcome of any sel of such local measurements is the square root of a determinant Since a 
determinant of order $N$ can be evaluated in a time which scales polynomially with $N$, the quantum algoithm can be simulated efficiently on a classical computer This is the no-go theorem mentioned in the introduction

We now add measurements of the local charge $Q_{l}=$ $n_{\imath \uparrow}+n_{t \downarrow}$ to the algorithm The elgenvalues of $Q_{1}$ are $0,1,2$ The probability that cha1ge one is measuied is given by the expectation value of the projection operator

$$
P_{l}=1-\left(1-Q_{l}\right)^{2}=a_{i \uparrow}^{\dagger} a_{l \uparrow} a_{l \downarrow} a_{t \downarrow}^{\dagger}+a_{l \downarrow}^{\dagger} a_{l \downarrow} a_{l \uparrow} a_{l \uparrow}^{\dagger}
$$

The operator $P_{1}$ is the sum of two local operators in the computational basis The probability that $M$ spatial modes are singly occupred therefore consists of a sum of an exponentially large number $\left(2^{M}\right)$ of deter minants, so now a classical simulation need no longei scale polynomially with the number of modes Notice that a measurement of $Q_{1}$ contains less infor mation about the state than separate measurements of $n_{\imath \uparrow}$ and $n_{\imath \downarrow}$ The fact that partial measurements can add computational power is a basic pinciple of quantum algoithms [1]

Let us now see how these formal considerations could be implemented, by consti ucting a CNOT gate using only beam splitters, spin lotations, and chaige detectors To constiuct the gate we need one of two new bulding blocks that are enabled by charge detectors The first building block is the Bell-state analyzer shown in Fig 1 For this device it does not matter whether the charge detector operates as an electrometer or as a parity meter The second bu1ling block, shown in Fig 2, converts a charge parity measurement to a spin parity measurement We present each device in tuin and then show how to consti uct the CNOT gate

The Bell-state analyzer makes it possible to teleport [19] the spin state $\alpha|\uparrow\rangle+\beta|\downarrow\rangle$ of election $A$ to anothes

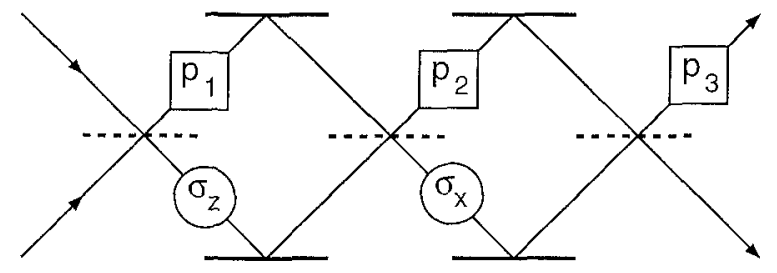

FIG 1 Bell state analyzer for nonmteracting elections, consisting of thiee 50/50 bcam splitters (dashed hoi izontal lines), lour miliols (solid holizonal lines), two local spm rotations (Paull matices $\sigma_{1}$ and $\sigma_{7}$ ), and thiee charge detectors (squares) The charge detectors may opciate erther as electrometers (counting the occupation $q_{1}=012 \mathrm{~m}$ an atm) ol as parily meters (measuning $p_{t}=q_{1} \bmod 2$ ) The first charge detector can identify the spin singlet state $\left|\Psi_{0}\right\rangle$, which is the only one of the foul Bell states $(2)-(4)$ to show $\left(p_{1}=0\right)$ Since $(\mathbb{1} \otimes$ $\sigma)\left|\Psi_{1}\right\rangle=-\left|\Psi_{0}\right\rangle$ the second chatge detector can identify $\left|\Psi_{1}\right\rangle$ when $p_{2}=0$ Finally since $\left(\mathbb{1} \otimes \sigma_{1} \sigma\right)\left|\Psi_{2}\right\rangle=\left|\Psi_{0}\right\rangle$ the thind change detectos can identify the two remanning states $\left|\Psi_{2}\right\rangle\left(\right.$ when $\left.p_{3}=0\right)$ and $\left|\Psi_{3}\right\rangle\left(\right.$ when $\left.p_{3}=1\right)$ election $A^{\prime}$, using a this election $B$ that is entangled with $A^{\prime}$ The telepoitation is peifor med by measuing the joint state of $A$ and $B$ in the Bell basis

$$
\begin{aligned}
& \left|\Psi_{0}\right\rangle=(|\uparrow \downarrow\rangle-|\downarrow \uparrow\rangle) / \sqrt{2}, \\
& \left|\Psi_{1}\right\rangle=(|\uparrow \downarrow\rangle+|\downarrow \uparrow\rangle) / \sqrt{2}, \\
& \left|\Psi_{2}\right\rangle=(|\uparrow \uparrow\rangle+|\downarrow \downarrow\rangle) / \sqrt{2}, \\
& \left|\Psi_{3}\right\rangle=(|\uparrow \uparrow\rangle-|\downarrow \downarrow\rangle) / \sqrt{2}
\end{aligned}
$$

A no-go theorem $[20,21]$ says that such a Bell measurement cannot be done deteiministically (meaning with $100 \%$ success piobability) without using interactions between the qubits Howevel, it has been noted that this theorem does not apply to qubits that possess an add1tronal degiee of fieedom [22], and that is how we will work alound it

In Fig 1 we show how a deterministic Bell measuiement for fermions can be performed using thiee 50/50 beam splitters, thiee charge detectors, and two local spin otations (represented by Pauli matices $\sigma$, and $\sigma$ ) The beam splitter scatters two elections into the same aim (bunching) if they are in the singlet state (2), and into two different arms (antibunching) if they are in one of the t1iplet states (3)-(5) (This can be easily undeistood [23] fiom the antisymmetiy of the wave function under pa1ticle exchange, demanded by the Pauli pinciple The singlet state is antisymmetic in the spin degiee of fieedom, so the spatial pait of the wave function should be

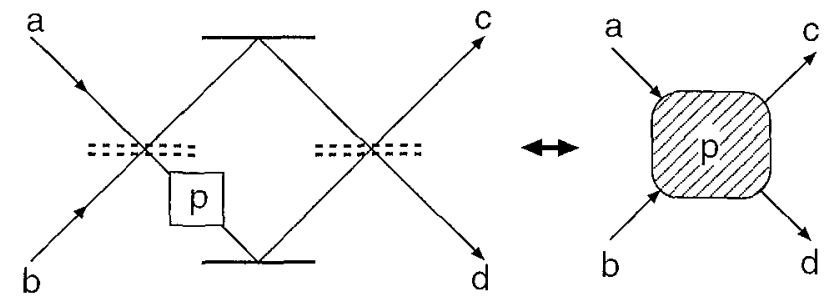

FIG 2 Gate that conver ts a charge party measurement to a spin paily measurement The shaded box at the ingt iepiesents the cucuit shown at the left A pail of elections is incident in aı ms $a$ and $b$ A polarızıng beam splıtter (double dashed line) transmits spin up and reflects spin down A charge detector recolds bunching $(p=0)$ or antibunching $(p=1)$ and passes the elections on to a second polarizing beam splitter If each election at the input is in a spin etgenstate $|\uparrow\rangle$ or $|\downarrow\rangle$, then output equals input and $p$ measures the spm paity ( $p=1$ if the two spins axe aligned, and $p=0$ if they are opposite) The gate can be used to encode a qubil $|\uparrow\rangle$ as the two particle state $|\uparrow\rangle|\uparrow\rangle$ and $|\downarrow\rangle$ as $|\downarrow\rangle|\downarrow\rangle$ For that pur pose the input consists of the qubit to be encoded in ai $m a$ plus an ancilla in arm $b$ in the state $(|\uparrow\rangle+|\downarrow\rangle) / \sqrt{2}$ The output is the requised two-particle state in alms $c$ and $d$ lor $p=1$ For $p=0$ it becomes the requiled state after a spm-flip $\left(\sigma_{1}\right)$ operdtion on the election in al m d 
symmetric, and vice versa for the ti plet state) Let $p_{t}$ be the charge $q_{l}$ measured by detector $l, \bmod 2$ So $p_{l}=0$ means bunching and $p_{t}=1$ means antibunching afteı bedm splittei $l$ The quantity

$$
\mathcal{B}=p_{1}+p_{1} p_{2}+p_{1} p_{2} p_{3}
$$

takes on the value $0,1,2$, or 3 depending on whether the incident state is $\left|\Psi_{0}\right\rangle,\left|\Psi_{1}\right\rangle,\left|\Psi_{2}\right\rangle$, or $\left|\Psi_{3}\right\rangle$, respectively The measurement of $\mathcal{B}$ is therefore the required projective measuiement in the Bell basis It is a destructive measurement, so it does not matter whether the charge detector operates as an electrometer (measuring $q_{l}$ ) or as a paisty meter (measuing $p_{l}$ )

In Fig 2 we show how a charge detector operating as a pailty meter can be used to measure in a nondestiuct1ve way whether two spins are the same or opposite "Nondestiuctive" means without measuing whether the spin is up or down The device consists of two pola1izing beam splitteis in selies, with the chaige detector in between (A polarizing beam splitter fully transmits $\uparrow$ and fully reflects $\downarrow$ ) At the input two elections are incident in different aims Input equals output if each election is in a spin eigenstate The measuied chaige parity then records whether the two spins are the same o1 opposite We will refer to this device as an encoder, because it can deteiministically entangle a qubit in the arbitiaiy state $\alpha|\uparrow\rangle+\beta|\downarrow\rangle$ and an ancilla in the fixed state $(|\uparrow\rangle+|\downarrow\rangle) / \sqrt{2}$ into the two-particle entangled state $\alpha|\uparrow\rangle|\uparrow\rangle+\beta|\downarrow\rangle|\downarrow\rangle$

To const 1 uct a CNOT gate using the Bell-state analyze we follow Ref [2], where it was shown that teleportation can be used to convert a probabilistic logical gate into a nearly deteiministic one It is well known that a piobabilistic CNOT gate can be constiucted from beam splitters and single-qubit operations The design of Pittman et al [7] has success probability $\frac{1}{4}$ and works for fermions as well as bosons It consumes an entangled pan of ancillas, which can be created probabilistically using a beam splitter and charge detector [14] Because the gate is not deterministic, 11 cannot be used in a scalable way inside the computation However, the CNOT gate can be repeatedly executed off-line, independent of the progiess of the quantum algoithm, until it has succeeded Two Bell measurements telepor the CNOT operation into the computation [24], when needed In this way a quantum algo11 thm can be executed using only single-paiticle Hamiltonians and single-particle measurements

In Fig 3 we show how to construct a CNOT gate using the encoder Oui design was inspiled by that of Pittman ef al [7], but rather than being probabilistic it is exactly deterministic We take two encoders in selies, with a change of basis on going fiom the first to the second encoder The change of basis is the Hadamaid transior mation

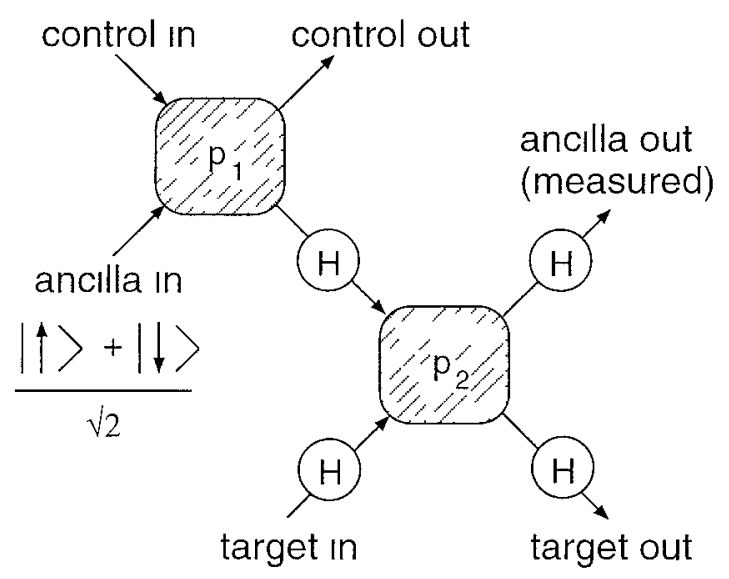

FIG 3 Deter minıstic CNOT gate for noninteracting electrons Each shaded box contans a pail of polanizing beam splitters and a charge detector, as described in Fig 2 The four Hadamaid gates $H=\left(\sigma_{1}+\sigma\right) / \sqrt{2}$ lotate the spins entering and leaving the second box The input of the CNOT gate consists of the contiol and target qubits plus an ancilla in the state $(|\uparrow\rangle+|\downarrow\rangle) / \sqrt{2}$ The spin of the ancilla is measured at the output. The outcome of that measurement together with the two pailties $p, p$, measured by the charge detectors determine which operations $\sigma_{c} \sigma_{t}$ one has to apply to control and target at the outpul in order to complete the CNOT operation For the contiol, $\sigma_{c}=\sigma_{z}$ if $p_{2}=0$ while $\sigma_{t}=\mathbb{1}$ if $p_{2}=1$ For the taiget, $\sigma_{t}=\sigma_{1}$ if the ancilla is down and $p_{1}=1$, ol if the ancilla is up and $p_{1}=0$ Otherwise, $\sigma_{t}=\mathbb{1}$ The calcu lation is given in Ref [30]

$$
|\uparrow\rangle \rightarrow(|\uparrow\rangle+|\downarrow\rangle) / \sqrt{2}, \quad|\downarrow\rangle \rightarrow(|\uparrow\rangle-|\downarrow\rangle) / \sqrt{2}
$$

The CNOT operation flips the spin of the taiget qubit if the spin of the contiol qubit is $\downarrow$ Cont1ol and taiget are input into separate encoders The ancrlla of the encoder for the contiol is fed back into the encoder for the taiget At the output, the spin of the ancilla is measured Conditioned on the outcome of that measuiement and on the two parities measured by the encoders, a Paul matı $1 x$ has to be applied to contiol and taiget to complete the CNOT operation

The computational power of the parity detectors is remarkable The CNOT gate of Fig 3 requires a single ancilla to achreve a $100 \%$ success probability, while the optimal design of LOQC needs $n$ ancillas in a specially prepared entangled state for a $1-1 / n^{2}$ success probability [8] In this respect it would seem that FEQC is computationally mote powerful than LOQC, but we emphasize that $F_{1} g$ applies to bosons as well as termions If parity detectors could be realized for photons (and there exist pioposals in the literatuie [6]), then the design of Fig 3 would diamatically simplify existing schemes for LOQC

In conclusion, we have shown that fiee-election quan tum computation (FEQC) is possible in pinciple either nearly deterministically (using a Bell-state analyzer with 
a cha1ge detector operating as an elect1ometer) or exactly deterministically (using an encoder with a charge detector operating as a parity meter) Unlike photons, elections interact stiongly if brought close together, so there is no need to rely exclusively on single-paiticle Hamiltonians We expect that FEQC would ultimately be used for flying qubits [25], while other gate designs based on shoit-1ange interactions [15,26] would be prefer ied for stationary qubits

The two ingredients of the cricurts considered here, beam splitters [27,28] and cha1ge detectors $[13,16,17]$, have both been realized by means of point contacts in a two-dimensional election gas The time-1esolved detection required for the operation as a logical gate has not yet been realized The curiently achievable time iesolution for charge detection is $\mu \mathrm{s}$ [16], while the resolution required for ballistic elections in a semiconductor is in the ps 1ange That time scale is not 1naccessible [29], but it might not be possible to reach the required single-election sensitivity due to the unavordable shot noise in the charge detector In the light of this, is could be more practical to stait with isolated elections in an aisay of quantum dots, lather than with flying qubits, in oider to investigate the potential and limitations of our theoretical concept on a presently accessible time scale

We have benefitted from discussions with B M Terhal This work was suppoited by the Dutch Science Foundation NWO/FOM, by the US Aimy Research Office (Giants No DAAD 19-02-0086 and No DAAD 19-01-C-0056), and by the Cambi1dgeMIT Institute, Ltd

' Peımanent addıess IBM, T J Watson Reseaıch Center, PO Box 218, Yoiklown Heights, NY 10598, USA

[1] M A Nielsen and I L Chuang, Quantum Computation and Quantum Information (Cambirdge Universsty, Cambi idge, 2000)

[2] E Knill, R Laflamme, and G J Milburn, Natu1c (London) 409, 46 (2001)

[3] B M Terhal and D P DiVincenzo, Phys Rev A 65, 032325 (2002)

[4] E Knill, quant-ph/0108033

[5] M Koashi, T Yamamoto, and N Imoto, Phys Rev A 63, 030301 (2001)
[6] D Gottesman, A Kilacv, and J Preskill, Phys Rev A 64, $012310(2001)$

[7] T B Pittman, B C Jacobs, and J D Fianson, Phys Rev A 64,062311(2001)

[8] I D Fianson, M M Donegan, M J Fitch, B C Jacobs, and T B Pittman, Phys Rev Lell 89, 137901 (2002)

[9] H F Holmann and S Takeuchı, Phys Rev A 66, 024308 (2002)

[10] T C Ralph, N K Langford, T B Bell, and A G White, Phys Rev A 65, 062324 (2002)

[11] J L Dodd, T C Ralph, and G J Milbuin, Phys Rev A 68, $042328(2003)$

[12] J D Fianson, B C Jacobs, and T B Pittman, quant-ph/ 0401133

[13] E Buks, R Schuster, M Herblum, D Mahalu, and V Umansky, Natu1e (London) 391, 871 (1998)

[14] S Bose and D Home, Phys Rev Lett 88, 050401 (2002)

[15] D Loss and D P Divincenzo, Phys Rev A 57, 120 (1998)

[16] J M El cciman, R Hanson, L H Willems van Beveren, L M K Vandessypen, and L P Kouwenhoven, Appl Phys Lett 84, 4617 (2004)

[17] $M$ Field, C G Smith, M Peppe1, D A Rilchie, J E F Fiost, G A C Jones, and D G Hasko, Phys Rev Lett. 70, 1311 (1993)

[18] S B Biavyı and A Yu Kitaev, quant-ph/0003137

[19] C H Bennctl, G Biassard, C Crepeau, R Jossa, A Peles, and W K Wootless, Phys Rev Lett 70, 1895 (1993)

[20] L Valdman and N Yolan, Phys Rev A 59, 116 (1999)

[21] N Lutkenhaus, J Calsamiglia, and K-A Suominen, Phys Rev A 59, 3295 (1999)

[22] P G Kwiat and H Wenfuler, Phys Rev A 58, 2623(R) (1998)

[23] G Burkald, D Loss, and E V Sukhor ukov, Phys Rev B 61, 16303(R) (2000)

[24] D Gottesman and I L Chuang, Natu1e (London) 402 , $390(1999)$

[25] A E Popescu and R Ioniciolu, cond-mal/0306401

[26] B E Kane, Nature (London) 393, 133 (1998)

[27] M Henny, S Oberholzer, C Stıunk, T Heinzel, $\mathrm{K}$ Ensslin, M Holland, and C Schonenberger, Scrence 284, 296 (1999)

[28] W D Olıvel, J Kım, R C Liu, and Y Yamamoto, Science 284, 299 (1999)

[29] E A Shaner and S A Lyon, cond-mat/0308460

[30] C W J Beenakkeı, D P DiVincenzo, C Emary, and M Kindeı mann, quant-ph/0401066 (see append $1 \mathrm{x}$ ) 